\title{
H. pylori infection-induced MSC differentiation into CAFs promotes epithelial-mesenchymal transition in gastric epithelial cells
}

\author{
QIANG ZHANG $^{1 *}$, MEI WANG ${ }^{1 *}$, FENG HUANG $^{1}$, TINGTING YANG $^{1}$, JIE CAI $^{1}$, \\ XU ZHANG ${ }^{1}$, WEI ZHU ${ }^{1}$, HUI QIAN ${ }^{1}$ and WENRONG XU ${ }^{1,2}$
}

${ }^{1}$ Key Institute of Clinical Laboratory Science, School of Medical Science and Laboratory Medicine, Jiangsu University,

${ }^{2}$ Affiliated Hospital of Jiangsu University, Zhenjiang, Jiangsu 212013, P.R. China

Received July 24, 2013; Accepted October 10, 2013

DOI: $10.3892 /$ ijmm.2013.1532

\begin{abstract}
Mesenchymal stem cell (MSC) tropism to injured tissue sites in response to inflammation and wounds has been suggested. MSC activation and recruitment by Helicobacter pylori (H. pylori)-infected gastrointestinal epithelial cells has been demonstrated. As a component of the chronic gastritis microenvironment, MSCs play critical roles in the development of $\mathrm{H}$. pylori-associated gastric mucosal lesions/malignancies. However, the mechanisms responsible for this process remain largely unknown. In this study, we demonstrate that $H$. pylori infection induces the differentiation of MSCs into cancerassociated fibroblast (CAF)-like cells. H. pylori-infected MSCs possessed an altered cytokine profile and induced epithelialmesenchymal transition in gastric epithelial cells, leading to destroyed cell junctions, enhanced cell migration, reduced cell apoptosis and increased oncogenic potential. In conclusion, our findings indicate that $H$. pylori infection may cause gastric lesions/malignancies by inducing the differentiation of MSCs into CAFs and suggest a novel mechanism of action and role of MSCs in the development and progression of gastric cancer.
\end{abstract}

\section{Introduction}

Helicobacter pylori (H. pylori) infection-associated gastritis is one of the most common infectious diseases worldwide. Epidemiological, pathophysiological and histological evidence

Correspondence to: Professor Wenrong Xu or Professor Hui Qian, Key Institute of Clinical Laboratory Science, School of Medical Science and Laboratory Medicine, Jiangsu University, 301 Xuefu Road, Zhenjiang, Jiangsu 212013, P.R. China

E-mail: icls@ujs.edu.cn

E-mail: 1stmmmlst@163.com

*Contributed equally

Key words: human umbilical cord-mesenchymal stem cells, cancerassociated fibroblast, Helicobacter pylori, gastric cancer, epithelialmesenchymal transition has demonstrated that $H$. pylori infection-associated gastritis is a major cause of gastric cancer (1-3). Although the mechanisms by which $H$. pylori induces gastric lesions/malignancies have been extensively investigated, the role of the $H$. pylori infection-associated chronic inflammation microenvironment in this process is poorly understood.

Mesenchymal stem cells (MSCs) are multipotent stem cells that have been found in several different tissues (4-6). MSCs are able to migrate across tissues and differentiate into a variety of cells, depending on the surrounding microenvironment $(7,8)$. In addition to normal tissues, MSCs have been found in injured tissue sites, suggesting a potential for the application of MSCs in regenerative medicine. MSC tropism for inflammation and cancer sites has also been reported, which links MSCs to the development of inflammation-associated cancer (9-14). In addition, the $H$. pylori-induced epithelial response can direct the homing of MSCs into the gastric mucosa $(15,16)$. As a component of the chronic gastritis microenvironment, MSCs play critical roles in gastric carcinogenesis and progression; however, little is known about the mechanisms by which MSCs participate in this process.

Cancer-associated fibroblasts (CAFs), a cell population that exists in human carcinomas, play a tumor-promoting role $(11,12)$. CAFs that express fibroblast activation protein (FAP) and $\alpha$-smooth muscle actin ( $\alpha$-SMA) create a niche for cancer cells and promote cancer metastasis $(17,18)$. As the main cellular component of the tumor stroma, CAFs can also induce the epithelial-mesenchymal transition (EMT) of malignant cells and promote angiogenesis (19). MSCs have been identified as a major source for CAFs (18). The transition of MSCs into CAFs contributes to tumor progression, angiogenesis and metastasis (18). We have previously reported that co-culture with conditioned medium (CM) and microvesicles (MVs) from gastric cancer cells induces the differentiation of MSCs into CAFs (20).

To further demonstrate the role of MSCs in H. pyloriinduced gastritis and gastric cancer, in this study, we designed a human umbilical cord MSC (hucMSC)/H. pylori co-culture model and evaluated the biological effects of the infected hucMSCs on normal gastric epithelial cells. Our results demonstrated that $H$. pylori infection induced the expression of CAF 
markers and cytokines in the hucMSCs. The infected hucMSCs promoted GES-1 normal gastric epithelial cells to acquire a mesenchymal phenotype through the process of EMT. The infected hucMSCs inhibited proliferation and promoted the invasion of GES-1 cells through a paracrine mechanism. Our findings may enhance the understanding of the role of MSCs in $H$. pylori infection-associated gastric cancer.

\section{Materials and methods}

HucMSCs isolation and cell culture. HucMSCs were obtained as previously described (4). Fresh umbilical cords were collected from informed, consenting mothers and processed within $6 \mathrm{~h}$. The cords were rinsed twice in phosphate-buffered saline (PBS) containing penicillin and streptomycin and the cord vessels were removed. The washed cords were cut into sections of $1-3 \mathrm{~mm}^{2}$ in size and were allowed to float in DMEM containing 10\% fetal bovine serum (FBS) (Invitrogen, Carlsbad, CA, USA), $1 \%$ penicillin and streptomycin. The sections of the cords were subsequently incubated at $37^{\circ} \mathrm{C}$ in humidified air with $5 \% \mathrm{CO}_{2}$ and the medium was changed every 3 days after the initial plating. When well developed colonies of fibroblast-like cells reached $80 \%$ confluence, the cultures were trypsinized and passaged into new flasks for further expansion. The characteristics of the isolated hucMSCs, including morphological appearance, surface antigens, differentiation potential and gene expression were investigated as previously described $(4,21)$. It was confirmed that hucMSCs were obtained and the MSCs at passage 3 were used for the experiments. All experimental protocols were approved by the Ethics Committee of Jiangsu University, Zhenjiang, China.

Cell culture, H. pylori strain and growth conditions. Human immortalized GES-1 cells were purchased from Cowin Biotech Co., Ltd. (Beijing, China) and maintained in RPMI1640 medium (Invitrogen) supplemented with 10\% FBS at $37^{\circ} \mathrm{C}$ in a $5 \% \mathrm{CO}_{2}$ humidified atmosphere. The $H$. pylori strain 11673 was kindly provided by Dr Shi-He Shao (Jiangsu University). The $H$. pylori strain was grown in trypticase soy agar supplemented with $5 \%$ sheep blood and incubated at $37^{\circ} \mathrm{C}$ under a microaerophilic atmosphere. For the co-culture experiments, the $H$. pylori strain was grown for $48 \mathrm{~h}$, resuspended in DMEM supplemented with $10 \%$ FBS and adjusted to $\mathrm{OD}_{600 \mathrm{~nm}}=1$ [corresponding to $1 \times 10^{8}$ colony-forming units $(\mathrm{CFU}) / \mathrm{ml}]$ prior to infection.

Co-culture of hucMSCs with H.pylori. HucMSCs cells were trypsinized, resuspended in DMEM supplemented with $10 \%$ FBS and seeded into culture flask. Twelve hours after seeding, grown colonies of $H$. pylori $(48 \mathrm{~h}$ ) were collected and the bacterial cells were added to the monolayer at a multiplicity of infection (MOI) of 100 bacteria/cell. Cultures were maintained at $37^{\circ} \mathrm{C}$ under a $5 \% \mathrm{CO}_{2}$ humidified atmosphere for $24 \mathrm{~h}$. The culture supernatants were harvested, centrifuged for $5 \mathrm{~min}$ at $3,000 \mathrm{rpm}$, filtered through $0.45-\mu \mathrm{m}$ filter units, and stored at $-80^{\circ} \mathrm{C}$ until use. The treated cells were harvested at the indicated time and subjected to the following experiments. For the controls, uninfected hucMSCs (hucMSCs) were processed in a similar manner in the absence of bacteria. Three duplicate wells were prepared for each experimental condition.
Exposure of GES-1 cells to CM from H. pylori-infected hucMSCs. The GES-1 cells were harvested as described above and cultured in RPMI-1640 medium supplemented with $10 \%$ FBS and antibiotics. The GES-1 cells were exposed to freshly harvested CM from the uninfected hucMSCs or the H. pylori-infected hucMSCs for $48 \mathrm{~h}$. The control GES-1 cells were processed in a similar manner in RPMI-1640 medium supplemented with $10 \%$ FBS. All reactions were repeated 3 times independently to ensure the reproducibility.

RNA extraction and quantitative reverse transcription $P C R$ ( $q R T-P C R)$. Total RNA was extracted using TRIzol reagent (Invitrogen) and cDNA was synthesized using a reverse transcription kit according to the manufacturer's instructions (Toyobo, Osaka, Japan). The primers used in this study were produced by Invitrogen (Shanghai, China). qRT-PCR analysis was performed to detect the changes in the expression of FAP, $\alpha$-SMA, E-cadherin, N-cadherin and vimentin genes (RotorGene 6000 Real-Time PCR Machine; Corbett Life Science, Sydney, Australia). An endogenous 'housekeeping' gene ( $\beta$-actin) was quantified to normalize the results. The primers used in this study were as follows: FAP forward, 5'-ATA GCAGTGGCTCCAGTCTC-3' and reverse, 5'-GATAA GCCGTGGTTCTGGTC-3'; $\alpha$-SMA forward, 5'-ATAGCAG TGGCTCCAGTCTC-3' and reverse, 5'-GATAAGCCGTGG TTCTGGTC-3'; E-cadherin forward, 5'-CGCATTGCCACA TACACTCT-3' and reverse, 5'-TTGGCTGAGGATGGTGT AAG-3'; N-cadherin forward, 5'-AGTCAACTGCAACCGT GTCT-3' and reverse, 5'-AGCGTTCCTGTTCCACTCAT-3'; vimentin forward, 5'-GAGCTGCAGGAGCTGAATG-3' and reverse, 5'-AGGTCAAGACGTGCCAGAG-3'; and $\beta$-actin forward, 5'-CACGAAACTACCTTCAACTCC-3' and reverse, 5'-CATACTCCTGCTTGCTGATC-3'. All experiments were performed in triplicate.

Western blot analysis. The cells were collected and lysed in RIPA buffer (10 mM Tris-HCl, pH 7.4, $150 \mathrm{mM} \mathrm{NaCl,} 1 \mathrm{mM}$ EGTA, 0.1\% SDS, $1 \mathrm{mM} \mathrm{NaF,} 1 \mathrm{mM} \mathrm{Na} \mathrm{VO}_{4}, 1 \mathrm{mM}$ PMSF, $1 \mathrm{mg} / \mathrm{ml}$ aprotinin and $1 \mathrm{~g} / \mathrm{ml}$ leupeptin) on ice. Aliquots containing identical amounts of protein were fractionated by sodium dodecyl sulfate-polyacrylamide gel electrophoresis (SDS-PAGE) and then transferred onto methanol preactivated polyvinylidene difluoride (PVDF) membranes (Millipore, Billerica, MA, USA). The membranes were blocked by $5 \%$ w/v non-fat dry milk. Following sequential incubation with the primary and secondary antibody (Santa Cruz Biotechnology, Inc., USA), the signal was visualized using HRP substrate (Millipore) and analyzed using MD ImageQuant Software, as perviously described (20). The sources and dilution factors of the primary antibodies were as follows: rabbit polyclonal anti-FAP (1:500; Abcam, USA), anti-vimentin (1:2,000; Santa Cruz Biotechnology, Inc.), anti-N-cadherin (1:400; SAB; Signalway Antibody Co., Ltd., MD, USA), anti-E-cadherin (1:500), mouse monoclonal anti- $\alpha$-SMA (1:400), anti-BMI (1:400) (all from Bioworld Technology Inc., USA), anti-SOX2 (1:500), anti-Nanog (1:500) (both from Santa Cruz Biotechnology, Inc.), antiOct4 (1:400), anti-p53 (1:800), anti-p21 (1:1,000; all SAB; Signalway Antibody Co., Ltd.) and anti- $\beta$-actin $(1: 2,000$; Bioworld Technology Inc.). 
Luminex assay/ELISA. The concentrations of granulocytemacrophage colony-stimulating factor (GM-CSF), interleukin (IL)-6, IL-8, platelet-derived growth factor (PDGF), tumor necrosis factor (TNF)- $\alpha$, IL-10, IL-1 $\beta$, epidermal growth factor (EGF), IL-15, IL-17, IL-2, vascular endothelial growth factor (VEGF) and monocyte chemoattractant protein-1 (MCP-1) in the supernatants of the control (uninfected) and $H$. pylori-infected hucMSCs were measured using Luminex (Millipore)/ELISA (Dakewe Biotech, Ltd., Beijing, China) kits in accordance with the manufacturer's instructions.

Transwell invasion assay. The invasion assay was carried out as previously described (20). Briefly, GES-1 cells $\left(5 \times 10^{4}\right.$ cells $\left./ 200 \mu 1\right)$ suspended in serum-free medium were loaded into the upper compartment of a Transwell chamber and $600 \mu \mathrm{l}$ of $10 \%$ FBS-DMEM medium containing hucMSCs ( $5 \times 10^{4}$ cells/well) in the presence or absence of H.pylori (MOI, 100:1) were added to the bottom well of the Transwell chamber (Corning, Inc. Life Sciences, MA, USA). Following culture at $37^{\circ} \mathrm{C}$ in a humidified atmosphere of $5 \% \mathrm{CO}_{2}$ for $8 \mathrm{~h}$, the cells in the upper membrane were wiped with a wet Q-tip. The cells that had migrated through the membrane ( $8 \mu \mathrm{m}$ pore size) were fixed with $4 \%$ paraformaldehyde and stained with crystal violet. The cells were observed under a microscope and at least 10 fields of cells were assayed for each group. Each assay was repeated 3 times.

Cell apoptosis assay. Cell apoptosis was evaluated using the FITC-Annexin V Apoptosis Detection kit I (BioVision Inc. San Francisco, CA, USA). The GES-1 cells were harvested at $48 \mathrm{~h}$ after co-culture with CM from the infected and uninfected hucMSCs. The fractions of viable, necrotic and apoptotic cells were detected and quantified by flow cytometry.

Cell proliferation assay. The GES-1 cells were plated in 96-well plates $\left(5 \times 10^{3}\right.$ cells/well) and incubated at $37^{\circ} \mathrm{C}$ in a humidified atmosphere with $5 \% \mathrm{CO}_{2}$ for $12 \mathrm{~h}$. The cells were then treated with $\mathrm{CM}$ from the infected and uninfected hucMSCs and incubated for 4 days. At the indicated time points $(0,24,48,72$ and $96 \mathrm{~h})$, the absorbance of the samples was measured using a VersaMax Microplate Reader (Molecular Devices, LLC, Sunnyvale, CA, USA) at wavelength of $450 \mathrm{~nm}$. Each experiment was conducted in triplicate and repeated twice independently.

Cell colony-forming assay. The GES-1 cells plated in 6-well plates $\left(1 \times 10^{3}\right.$ cells/well) were incubated with DMEM (control), or CM from the uninfected hucMSCs or the H.pylori-infected hucMSCs for $48 \mathrm{~h}$, and then all groups were incubated at $37^{\circ} \mathrm{C}$ in a humidified atmosphere with $5 \% \mathrm{CO}_{2}$ for 15 days with RPMI-1640 medium with $10 \%$ FBS (normal medium). The medium was changed every 3 days. The number of colonies was then evaluated by crystal violet staining. The results are the mean values of 3 experiments and 3 replicate plates.

Statistical analyses. All data are expressed as the means \pm SD. SPSS software was used to analyze the data. The means of different treatment groups were compared by two-way ANOVA or the Student's t-test. A P-value $<0.05$ was considered to indicate a statistically significant difference.

\section{Results}

H. pylori infection promotes the differentiation of hucMSCs into CAFs. To determine the effects of $H$. pylori infection on the phenotype of hucMSCs, we infected the cells with $H$. pylori at an MOI of 100:1. The hucMSCs acquired a spindle-shaped morphology at $24 \mathrm{~h}$ after $H$. pylori infection (Fig. 1A). An increased expression of fibroblastic proteins $(\alpha$-SMA, FAP, vimentin and N-cadherin) has been defined as a marker for CAFs $(17,18)$. Thus, we detected the expression of CAF markers in the H. pylori-infected hucMSCs by qRT-PCR. The results revealed that $H$. pylori infection increased the expression of FAP, $\alpha$-SMA, N-cadherin and vimentin genes in the hucMSCs (Fig. 1B). In agreement with the gene expression data, FAP, $\alpha$-SMA, N-cadherin and vimentin protein levels were also increased in the hucMSCs upon exposure to H. pylori (Fig. 1C).

$H$. pylori infection induces the production of CAF-associated factors in hucMSCs. The upregulation of specific factors (IL-6, IL-8, VEGF and EGF) has been suggested as another marker for CAFs $(13,18,22)$. To that end, we detected CAF-associated functional factors in $H$. pylori-infected hucMSCs by Luminex assay. The results revealed that the expression of several cytokines and chemokines, such as IL-6, IL-8 and PDGF was markedly increased in the hucMSCs infected with $H$. pylori (Fig. 2A). The induction of IL-6, IL-8 and PDGF expression in the H.pylori-infected hucMSCs was further validated by ELISA (Fig. 2B). These data indicate that $H$. pylori infection induces the production of CAF-associated functional factors in MSCs.

H. pylori-infected hucMSCs induce EMT in GES-1 cells. To determine the effects of $H$. pylori-infected hucMSCs on gastric epithelial cells, we observed morphological changes in the control GES-1 cells, those cultured with $H$. pylori (11637; MOI, 100:1), and those cultured with uninfected and H. pylori-infected hucMSCs. The morphological shift from an epithelial to a fibroblastic phenotype was observed in the GES-1 cells treated with CM from both the uninfected and $H$. pylori-infected hucMSCs. However, the $H$. pylori infection of the hucMSCs significantly enhanced their ability to induce the acquirement of a fibroblastic phenotype in GES-1 cells (Fig. 3). We then analyzed the expression of EMT markers, including E-cadherin, $\mathrm{N}$-cadherin and vimentin, in the GES-1 cells that were treated with CM from the uninfected and $H$. pylori-infected hucMSCs. The results revealed that GES-1 cells exhibited a mesenchymal phenotype characterized by an impaired E-cadherin expression and an increased expression of vimentin and N-cadherin (Fig. 4).

H. pylori-infected hucMSCs enhance the invasive ability of GES-1 cells. EMT phenotypes are associated with an enhanced invasive ability. To determine whether $H$. pylori infection affects the pro-invasive capacity of the hucMSCs in GES-1 cells, an in vitro Transwell cell migration assay was performed (Fig. 5A). CM from the hucMSCs induced the migration of GES-1 cells. Compared with the control (control GES-1 cells) and hucMSC group, CM from the H. pyloriinfected hucMSCs induced a more aggressive phenotype in 
A

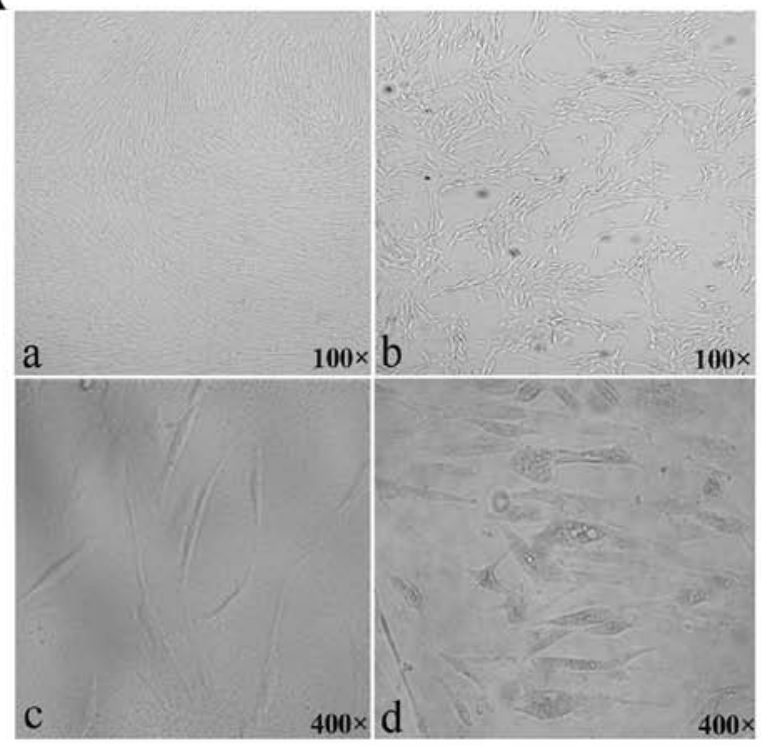

B
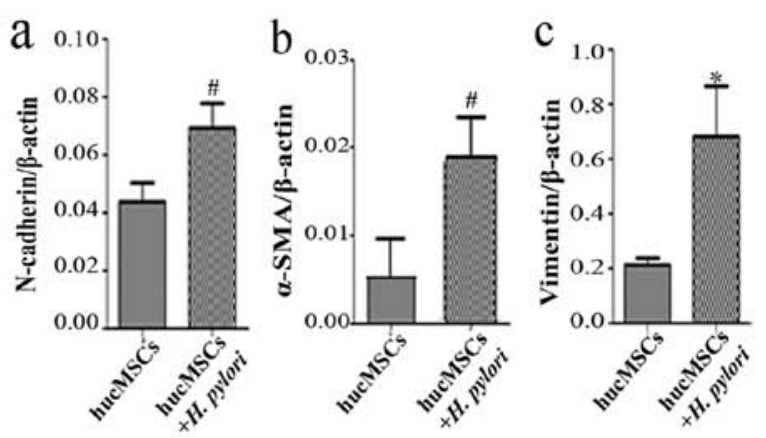

d

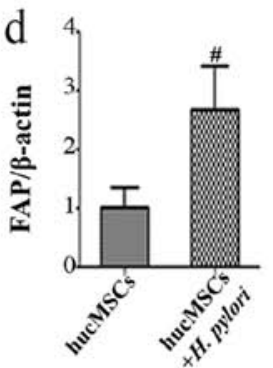

C

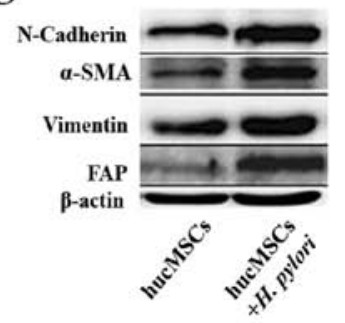

Figure 1. H. pylori infection triggers hucMSC differentiation into cancer-associated fibroblasts (CAFs). (A) Morphology of hucMSCs and hucMSCs infected with H.pylori: (a and c) hucMSCs; (b and d) hucMSCs infected with H. pylori (MOI, 100:1). (B) qRT-PCR analysis of CAF markers in $H$. pylori-infected hucMSCs: (a) N-cadherin; (b) $\alpha$-smooth muscle actin ( $\alpha$-SMA); (c) vimentin; and (d) fibroblast activation protein (FAP). (C) Western blot analysis of N-cadherin, $\alpha$-SMA, vimentin and FAP protein expression in $H$. pylori-infected hucMSCs. ${ }^{*} \mathrm{p}<0.01$ and ${ }^{\#} \mathrm{p}<0.05$, compared with the control group (uninfected cells) (n=3). MOI, multiplicity of infection.

A
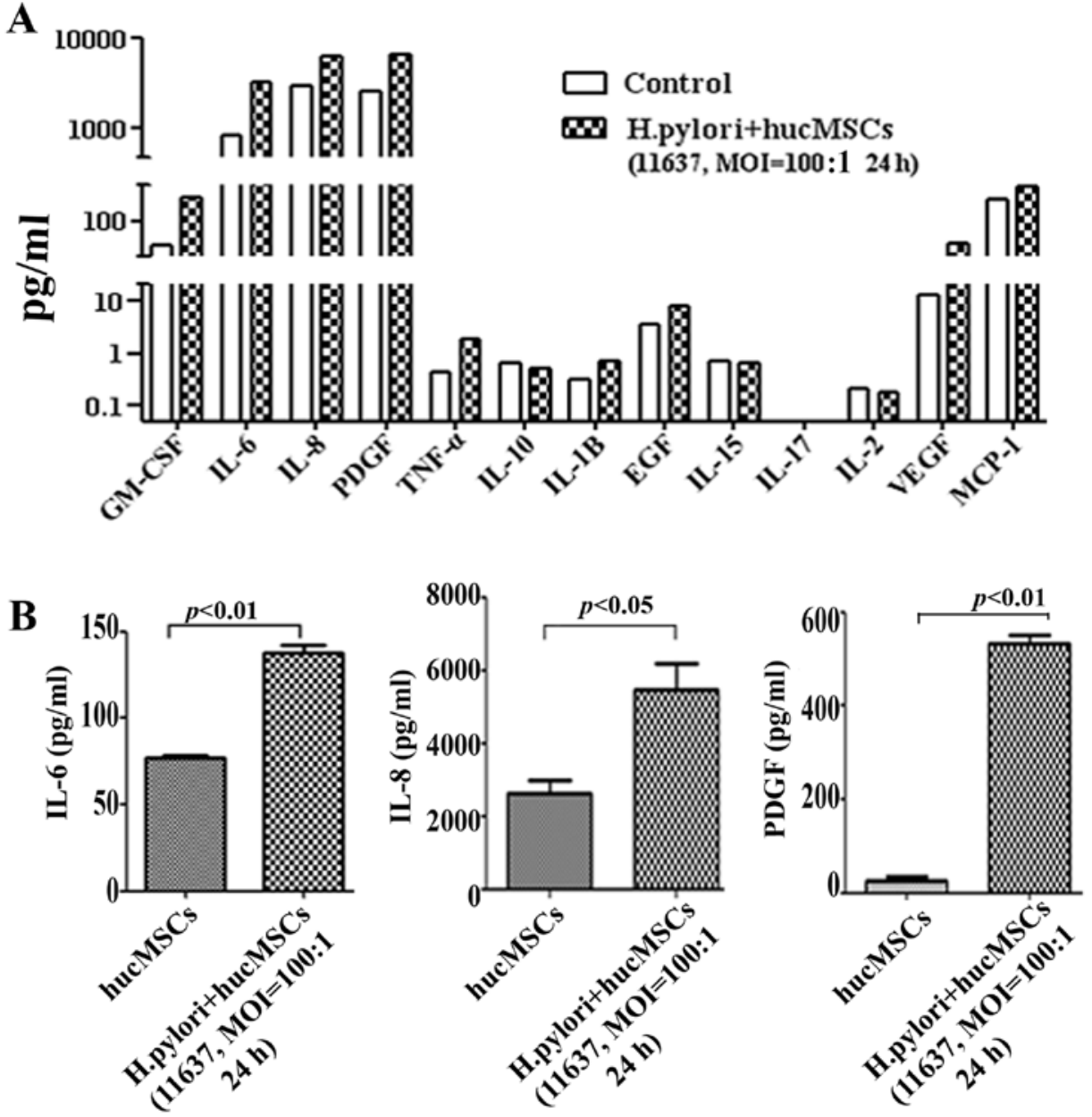

Figure 2. Cytokine profile of $H$. pylori-infected hucMSCs. (A) Luminex assay for the expression of cytokines in the conditioned medium from uninfected and $H$. pylori-infected hucMSCs. Results are expressed as fold increases compared with the uninfected cells. (B) Supernatants from uninfected and $H$. pyloriinfected hucMSCs were assessed for IL-6, IL-8 and PDGF expression by ELISA. Data are representative of 3 independent experiments. 


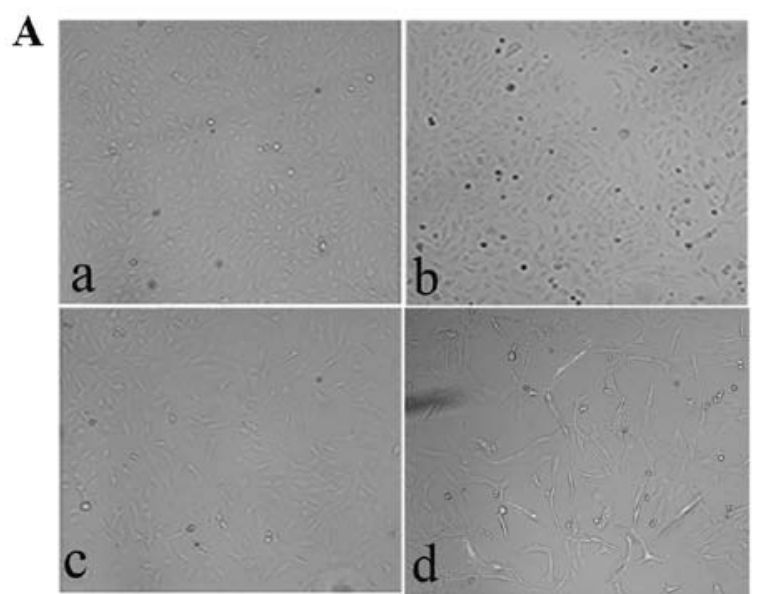

B

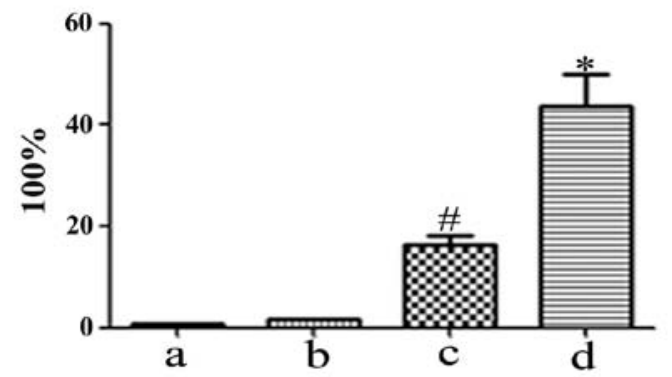

Figure 3. Morphological changes of gastric epithelial cells (GES-1) treated with conditioned medium (CM) from H. pylori-infected hucMSCs. (A) Morphology of GES-1 cells: (a) control cells; (b) GES-1 cells treated with H. pylori (11637, MOI, 100:1); (c) uninfected hucMSCs; and (d) H. pylori-infected hucMSCs GES-1 cells treated with CM from the infected hucMSCs displayed long and spindle-shaped fibroblasts. (B) Percentage of cells with morphological changes. These experiments were repeated 3 times. ${ }^{*} \mathrm{p}<0.01$ and ${ }^{\#} \mathrm{p}<0.05$, compared with the relative control group $(\mathrm{n}=3)$. MOI, multiplicity of infection.

A
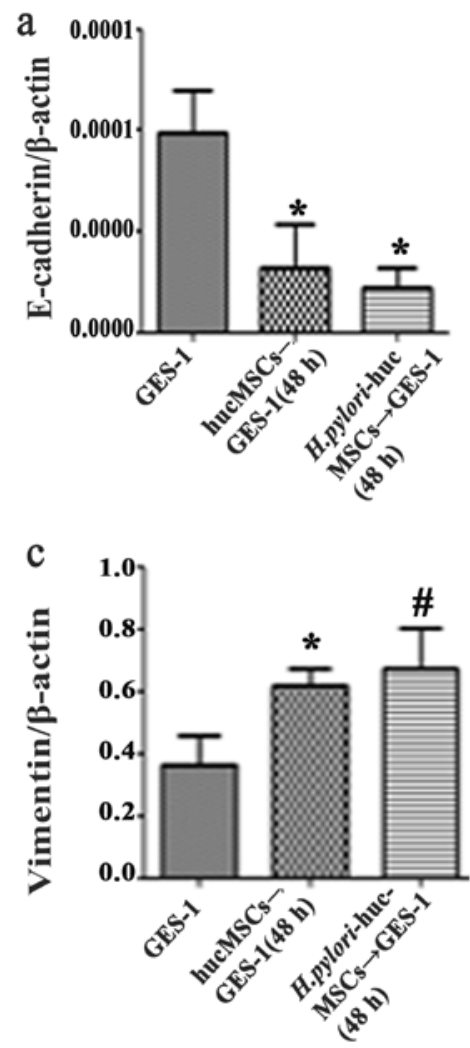

B

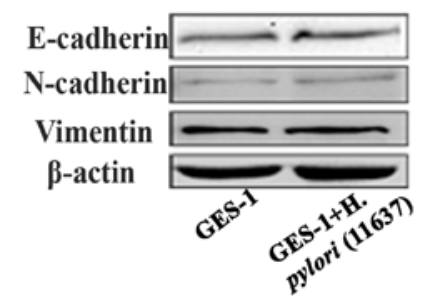

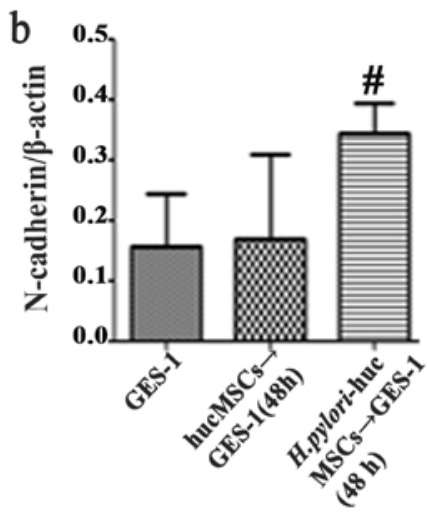

d

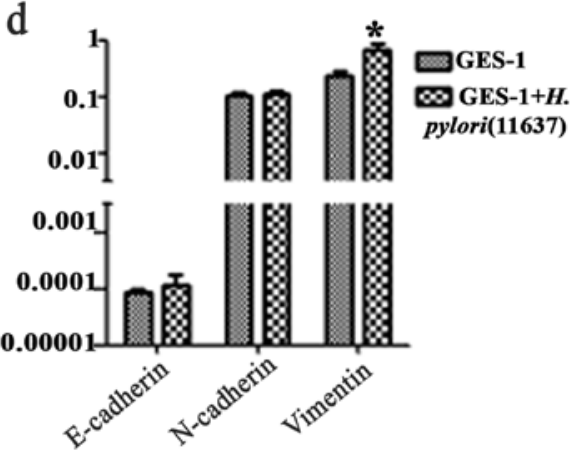

Figure 4. H. pylori-infected MSCs induce EMT in gastric epithelial cells. (A) qRT-PCR analysis of (a) E-cadherin; (b) N-cadherin; and (c) vimentin mRNA levels in control gastric epithelial cells (GES-1) and in GES-1 cells exposed to conditioned medium (CM) from uninfected and $H$. pylori-infected hucMSCs. (d) H. pylori increased the expression of E-cadherin and increased the expression of N-cadherin and vimentin at the mRNA and protein level in GES-1 cells. (B) Western blot analysis of E-cadherin, $\mathrm{N}$-cadherin and vimentin protein expression in the control GES-1 and those treated with CM from uninfected and H. pylori-infected hucMSCs. " $\mathrm{p}<0.05$ and ${ }^{\#} \mathrm{p}<0.01$, compared with the relative control group $(\mathrm{n}=3)$. 

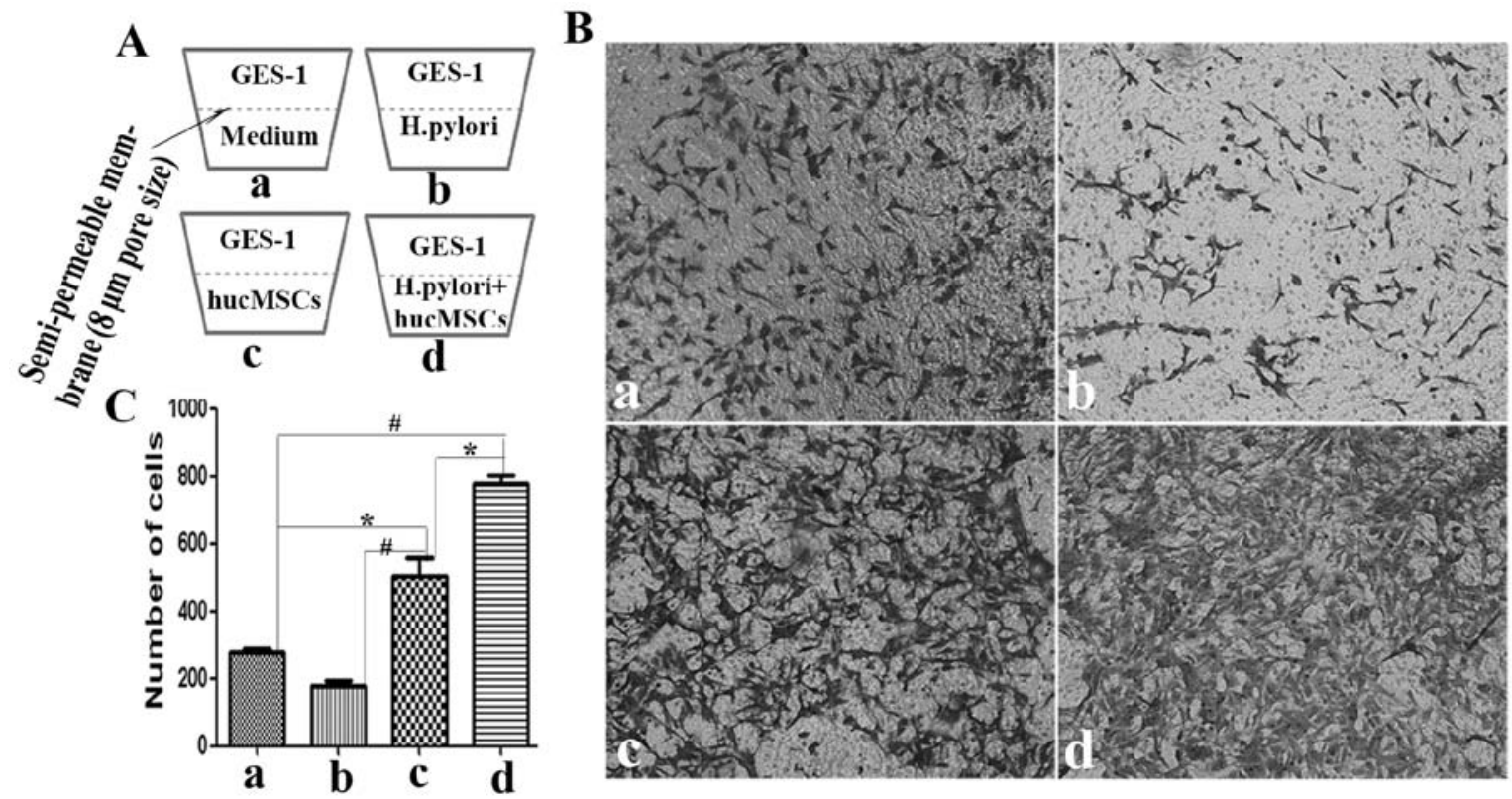

Figure 5. H. pylori-infected MSCs enhance the invasive ability of gastric epithelial cells. (A) The ability of control MSCs to induce gastric epithelial cell (GES-1) migration following $H$. pylori infection was evaluated by Transwell migration assay. (B) Representative images showing the migration of GES-1 cells in the infected and H. pylori-infected MSC group. Original magnification, x100. (a) Control GES-1 cells; (b) GES-1 cells treated with $H$. pylori; (c) uninfected hucMSCs; and (d) H.pylori-infected hucMSCs. (C) The number of migrated cells was quantified. $H$. pylori-infected hucMSCs more effectively enhanced GES-1 cell penetration through the membranes. The experiments were repeated 3 times. ${ }^{*} \mathrm{p}<0.05$ and ${ }^{\#} \mathrm{p}<0.01(\mathrm{n}=3)$.

the GES-1 cells (Fig. 5B). The number of migrated cells was then quantified (Fig. 5C).

H. pylori-infected hucMSCs reduce the apoptosis of GES-1 cells. The GES-1 cells were treated with CM from the H. pylori-infected hucMSCs for $48 \mathrm{~h}$, and cell apoptosis was analyzed by Annexin V-FITC/PI staining. The apoptotic rate significantly decreased following exposure to $\mathrm{CM}$ from the H. pylori-infected hucMSCs (5.06 vs. $0.43 \%, \mathrm{p}<0.05$ ) (Fig. 6A). We also detected the expression of apoptosis-related proteins in the GES-1 cells treated with CM from the $H$. pylori-infected hucMSCs. We found that the expression of Bax, a pro-apoptotic effector, decreased in the treated GES-1 cells, whereas the expression of the anti-apoptotic protein, Bcl-2, increased (Fig. 6B). These results indicated that the $H$. pylori-infected hucMSCs inhibited apoptosis in gastric epithelial cells.

H. pylori-infected hucMSCs inhibit the proliferation of GES-1 cells. The GES-1 cells were treated with CM from the $H$. pylori-infected hucMSCs for 4 days, and cell proliferation was analyzed by MTT assay. The results revealed that CM obtained from the infected hucMSCs significantly inhibited the proliferation of GES-1 cells in vitro (Fig. 6C).

H. pylori-infected hucMSCs induce stem cell properties in GES-1 cells. Several stem cell-related proteins, including SOX2, Nanog and BMI were detected in the GES-1 cells treated with CM from the H.pylori-infected hucMSCs. The H.pyloriinfected hucMSCs significantly upregulated the expression of SOX2, Nanog and BMI-1 in the GES-1 cells (Fig. 7A-a).

Effect of H. pylori-infected hucMSCs on the expression of oncoproteins and tumor suppressor proteins in GES-1 cells.
To determine whether $H$. pylori-infected hucMSCs can induce the transformation of GES-1 cells, we analyzed the expression of oncoproteins and tumor suppressor proteins in the GES-1 cells. The GES-1 cells were treated with CM derived from the H. pylori-infected hucMSCs for $48 \mathrm{~h}$. The results revealed that the $H$. pylori-infected hucMSCs increased the expression of mucin 2 (MUC2) and chemokine (C-X-C motif) receptor 4 (CXCR4) oncoproteins, and decreased the expression of the tumor suppressor proteins, p53 and p21, in the GES-1 cells (Fig. 7A-b).

H. pylori-infected hucMSCs stimulate the colony-forming ability of GES-1 cells. We further performed a cell colonyforming assay to assess the oncogenic potential of GES-1 cells that were treated with CM from the H.pylori-infected hucMSCs. Compared with the control GES-1 cells and those treated with $\mathrm{CM}$ from the uninfected hucMSCs, the cells treated with $\mathrm{CM}$ from the $H$. pylori-infected hucMSCs showed a significantly enhanced colony-forming ability (Fig. 7B and C).

\section{Discussion}

CAFs that express FAP and $\alpha$-SMA are key determinants in the malignant progression of cancer growth, vascularization and metastasis $(11,17,18)$. After being passaged successively 10 times in vitro without ongoing interaction with carcinoma cells, CAFs still retain their ability to promote tumor growth when co-injected with carcinoma cells into immunodeficient mice $(11,12)$. MSCs have been shown to be involved in H.pylori infection-associated gastric carcinogenesis (15). However, the mechanisms responsible for the promoting roles of MSCs in cancer initiation and progression are not yet well understood. In this study, we demonstrated that $H$. pylori infection induced 
$\mathbf{A}_{\text {. }}$
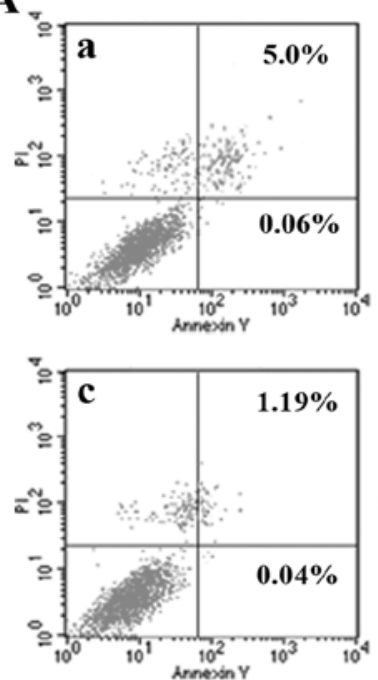
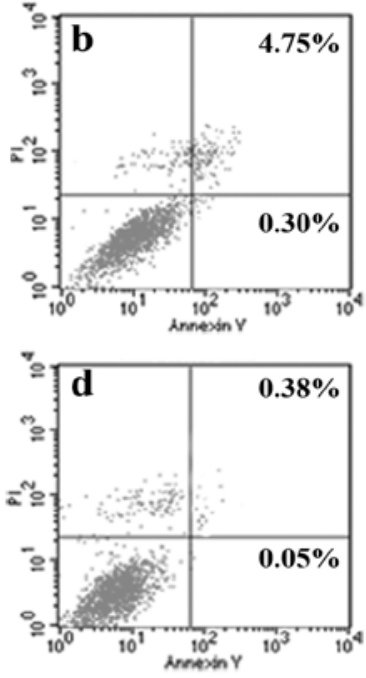

B
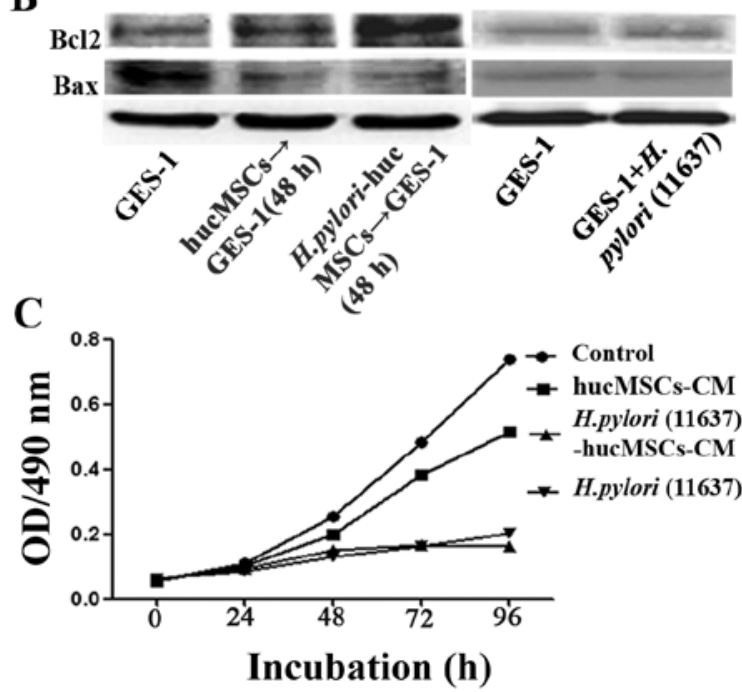

Figure 6. Effects of H. pylori-infected MSCs on the apoptosis and proliferation of gastric epithelial cells. (A) Flow cytometric assays of cell apoptosis in gastric epithelial cells (GES-1). At $48 \mathrm{~h}$ after co-culture with $H$. pylori 11637, conditioned medium (CM) from uninfected or $H$. pylori-infected hucMSCs, or GES-1 control cells was collected and stained with Annexin V/PI. The experiments were performed in triplicate. (a) GES-1 control cells; (b) GES-1 infected with H. pylori; (c) GES-1 incubated with CM from uninfected hucMSCs; (d) GES-1 incubated with CM from H. pylori-infected hucMSCs. "p<0.05 compared with untreated GES-1 cells. (B) Western blot analysis of Bcl-2 and Bax protein expression in GES-1 cells. (C) Growth curves of GES-1 cells treated with CM from GES-1 infected with H. pylori, and cells incubated with CM from uninfected hucMSCs or H. pylori-infected hucMSCs. The growth of GES-1 cells in the different groups was examined by MTT assay. GES-1 cells treated with medium only served as the controls.

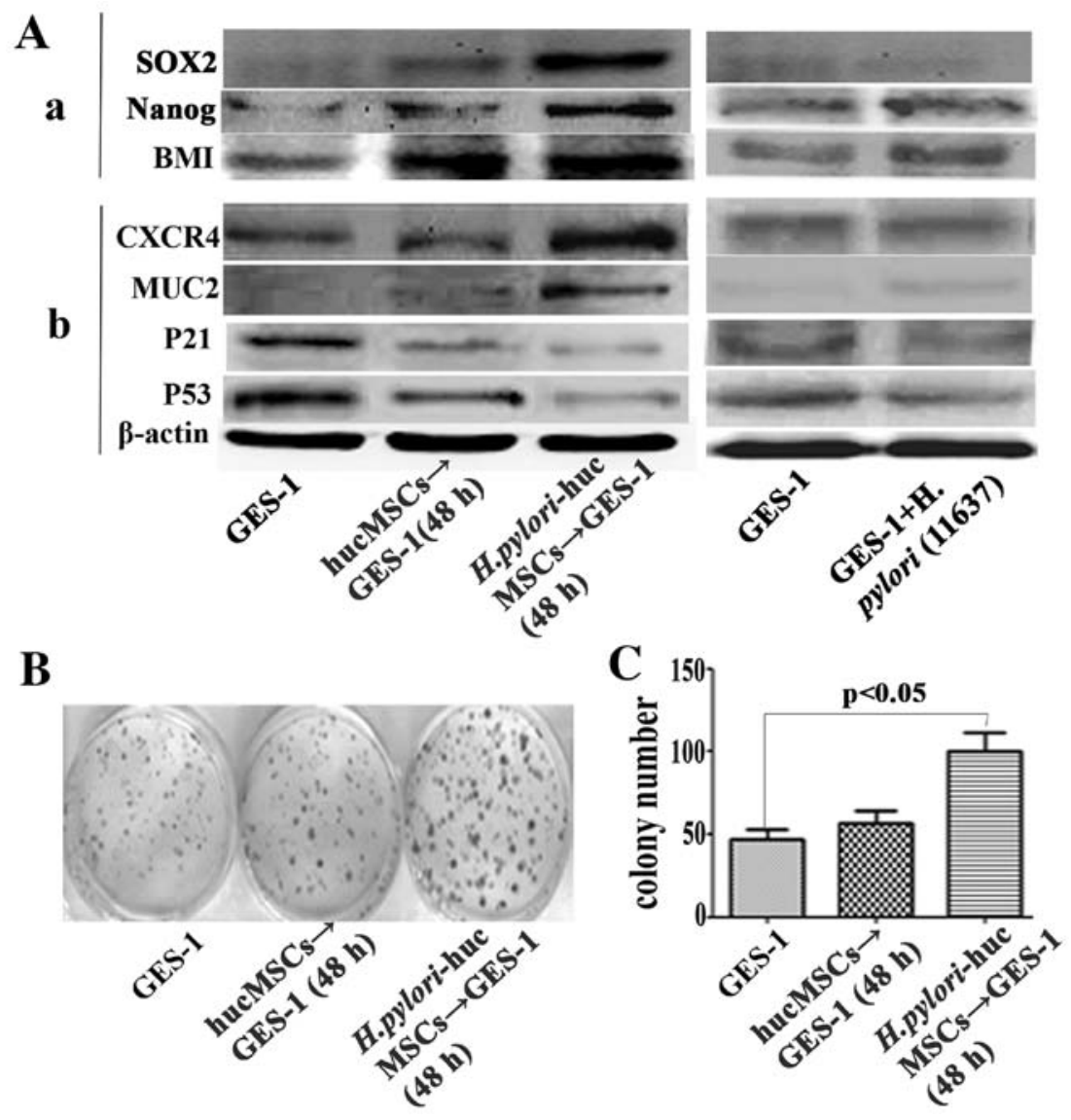

Figure 7. Effects of $H$. pylori-infected MSCs on stemness and cancer-related gene expression and colony-forming ability of gastric epithelial cells (A) (a) Western blot analysis of the expression of SOX2, Nanog and BMI proteins in gastric epithelial cells (GES-1) exposed to conditioned medium (CM) from control (GES-1) cells, uninfected and $H$. pylori-infected hucMSCs. (b) Western blot analysis of CXCR4, MUC2, p53 and p21 protein expression in GES-1 cells exposed to CM from control (GES-1) cells, uninfected and $H$. pylori-infected hucMSCs. (B) Cell colony-forming assay of GES-1 cells treated with CM from control (GES-1) cells, uninfected and $H$. pylori-infected hucMSCs. No colonies were formed in the GES-1 cells infected with $H$. pylori. (C) The number of formed colonies was quantified. 
typical CAF differentiation with the increased expression of CAF markers and cytokines in hucMSCs. Recent studies have demonstrated well-defined roles for CAF-associated cytokines, such as IL-6, IL-8, PDGF, VEGF, EGF and GM-CSF in tumorigenesis $(18,22,23)$. The results presented in this study suggest that $H$. pylori infection may induce the differentiation of MSCs into CAFs and enhance the secretion of multiple cytokines.

EMT is essential for the generation of new tissues during embryogenesis and plays pivotal roles in inflammation and wound healing $(24,25)$. EMT is defined as a biological process, in the course of which epithelial cell-cell adhesion is decreased by the downregulation of adhesion molecules, such as E-cadherin, and cell morphology becomes fibroblast-like with the upregulation of vimentin and N-cadherin (24). In addition to a loss in epithelial characteristics, EMT frequently coincides with the acquisition of motility and invasiveness, as well as an increase in the resistance to apoptosis and a markedly altered production of extracellular matrix components. Cancer is often viewed as the corrupted form of normal development (26-29). EMT has been implicated as a fundamental step of carcinogenesis (30) and represents one of the steps required for tumor progression through invasion and metastatic spread (31). We hypothesized that $H$. pyloriinfected MSCs may promote gastric lesions/malignancies under the conditions of chronic gastritis through the induction of EMT in gastric epithelial cells. To prove this hypothesis, we analyzed the phenotype of GES-1 cells co-cultured with CM from $H$. pylori-infected hucMSCs. We found that GES-1 cells exposed to CM from $H$. pylori-infected hucMSCs not only displayed a morphological shift from an epithelial to a fibroblastic phenotype, but also presented decreased E-cadherin expression, increased vimentin and $\mathrm{N}$-cadherin expression, and an enhanced invasive ability in vitro. These results are in agreement with data from previous studies, demonstrating that the loss of E-cadherin expression augments cellular dissemination and metastasis (32). These results support the hypothesis that $H$. pylori infection-induced MSC transition into CAFs results in gastric lesions/malignancies by promoting the occurrence of EMT.

Tumor development involves multiple steps and factors, including the activation of oncogenes, the inactivation of tumor suppressor genes, and the aberrant expression of apoptosis-related genes (33). In this study, we demonstrated that the infected hucMSCs enhanced the expression of the oncoproteins, MUC2 and CXCR4, whereas they inhibited the expression of the tumor suppressor proteins, p53 and p21, in GES-1 cells. We also demonstrated that the proliferation rate of the GES-1 cells was reduced by $\mathrm{CM}$ from $H$. pylori-infected hucMSCs. These results are consistent with those of other studies, suggesting that the proliferation rate of cancer cells decreases when the cells move and migrate $(34,35)$. Evidence suggests that cancer cells acquire stem cell-like properties through EMT (36). Mani et al (37) demonstrated an upregulation of stem cell markers through the induction of EMT in mammary epithelial cells and breast cancer cells. Additional studies have demonstrated that the induction of EMT not only promotes tumor cell invasion and metastasis, but also contributes to the acquisition of stem cell properties (38). In this study, we detected the expression of stem cell markers and evaluated the capacity of gastric epithelial cells to form colonies. We demonstrated that GES-1 cells expressed higher levels of the stemness markers, Nanog, BMI and SOX2, following exposure to CM from $H$. pylori-infected hucMSCs. Nanog, SOX2 and BMI have been shown to be critical factors for cancer initiation and progression (39-41). The upregulation of these stemness-related factors indicated the acquirement of stem cell properties in the GES-1 cells following exposure to CM from $H$. pylori-infected hucMSCs. In addition, the $H$. pyloriinfected MSCs markedly enhanced the colony-forming ability of gastric epithelial cells, suggesting that incubation with CM from $H$. pylori-infected MSCs endows gastric epithelial cells with both oncogenic potential and self-renewal ability. However, the specific factors that mediate this process and the signaling pathways involved remain to be identified.

Taken together, we demonstrate that $H$. pylori infection induces the differentiation of MSCs into CAF-like cells and that incubation with CM from $H$. pylori-infected MSCs destroys cell junctions, promotes cell invasion and enhances the colony-forming ability of gastric epithelial cells though the induction of EMT. Our findings suggest that $H$. pylori infection causes gastric lesions/malignancies by converting MSCs into CAFs, creating a unique microenvironment for the malignant transformation of the normal gastric epithelium. This study may aid in the understanding of the role and mechanisms of action of MSCs in the initiation and progression of $\mathrm{H}$. pyloriassociated gastric cancer.

\section{Acknowledgements}

This study was supported by the Major Research Plan of the National Natural Science Foundation of China (grant no. 91129718), the National Natural Science Foundation of China (grant nos. 81071421,81302119, 81000181 and 81201660), the Jiangsu Province Project of Scientific and Technological Innovation and Achievements Transformation (grant no. BL2012055), the Jiangsu Province Outstanding Medical Academic Leader and Sci-tech Innovation Team Program (grant no. LJ201117), the Doctoral Program Foundation of State Education Ministry (grant no. 20113227110011), the Jiangsu Province Natural Science Foundation (grant no. BK20130540) and the Jiangsu Province Department of Education Science Research Foundation (grant no. 13KJB320001).

\section{References}

1. Machado AM, Figueiredo C, Touati E, et al: Helicobacter pylori infection induces genetic instability of nuclear and mitochondrial DNA in gastric cells. Clin Cancer Res 15: 2995-3002, 2009.

2. Milne AN, Carneito F, O'Morain C and Offerhaus GJ: Nature meets nurture: molecular genetics of gastric cancer. Hum Genet 126: 615-628, 2009.

3. Chan AO, Chu KM, Huang C, et al: Association between Helicobacter pylori infection and interleukin 1beta polymorphism predispose to $\mathrm{CpG}$ island methylation in gastric cancer. Gut 56: 595-597, 2007.

4. Qiao C, Xu W, Zhu W, et al: Human mesenchymal stem cells isolated from the umbilical cord. Cell Biol Int 32: 8-15, 2008

5. Cao H, Xu W, Qian H, et al: Mesenchymal stem cell-like cells derived from human gastric cancer tissues. Cancer Lett 274: 61-71, 2009.

6. Schäffler A and Büchler C: Concise review: adipose tissuederived stromal cells - basic and clinical implications for novel cell-based therapies. Stem Cells 25: 818-827, 2007.

7. Charbord P: Bone marrow mesenchymal stem cells: historical overview and concepts. Hum Gene Ther 21: 1045-1056, 2010. 
8. Phinney DG and Prockop DJ: Concise review: mesenchymal stem/multipotent stromal cells: the state of transdifferentiation and modes of tissues repair - current views. Stem Cells 25: 2896-2902, 2007.

9. Sasaki M, Abe R, Fujita Y, Ando S, Inokuma D and Shimizu H: Mesenchymal stem cells are recruited into wounded skin and contribute to wound repair by transdifferentiation into multiple skin cell type. J Immunol 180: 2581-2587, 2008.

10. Spaeth E, Kloop A, Dembinski J, Andreeff M and Marini F: Inflammation and tumor microenvironments: defining the migratory itinerary of mesenchymal stem cells. Gene Ther 15 $730-738,2008$

11. Orimo A, and Weinberg RA: Stromal fibroblasts in cancer: a novel tumor-promoting cell type. Cell Cycle 5: 1597-1601, 2006.

12. Orimo A, Gupta PB, Sqroi DC, et al: Stromal fibroblasts present in invasive human breast carcinomas promote tumor growth and angiogenesis through elevated SDF-1/CXCL12 secretion. Cell 121: 335-348, 2005.

13. Glaire MA, EI-Omar EM, Wang TC and Worthley DL: The mesenchyme in malignancy: a partner in the initiation, progression and dissemination of cancer. Pharmacol Ther 136: 131-141, 2012.

14. Chamberlain G, Fox J, Ashton B and Middleton J: Concise review: mesenchymal stem cells: their phenotype, differentiation capacity, immunological features, and potential for homing. Stem Cells 25: 2739-2749, 2007.

15. Houghton J, Stoicov C, Nomura S, et al: Gastric cancer originating from bone marrow-derived cells. Science 306: 1568-1571, 2004.

16. Ferrand J,Lehours P, Schmid-Alliana A, Mégraud F and Varon C: Helicobacter pylori infection of gastrointestinal epithelial cells in vitro induces mesenchymal stem cell migration through an NF- $\kappa$ B-dependent pathway. PLoS One 6: e29007, 2011.

17. Dudás J, Fullár A, Bitsche M, et al: Tumor-produced, active interleukin-1 $\beta$ regulates gene expression in carcinoma-associated fibroblasts. Exp Cell Res 317: 2222-2229, 2011.

18. Spaeth EL, Dembinski JL, Sasser AK, et al: Mesenchymal stem cell transition to tumor-associated fibroblasts contributes to fibrovascular network expansion and tumor progression. PLoS One 4: e4992, 2009

19. Giannoni E, Bianchini F, Masieri L, Serni S, Torre E, Calorini L and Chiarugi P: Reciprocal activation of prostate cancer cells and cancer-associated fibroblasts stimulates epithelial- mesenchymal transition and cancer stemness. Cancer Res 70: 6945-6956, 2010

20. Gu J, Qian H, Shen L, et al: Gastric cancer exosomes trigger differentiation of umbilical cord derived mesenchymal stem cells to carcinoma-associated fibroblasts through TGF- $\beta / \mathrm{Smad}$ pathway. PLoS One 7: e52465, 2012.

21. Qian H, Yang $\mathrm{H}, \mathrm{Xu}$ W, et al: Bone marrow mesenchymal stem cells ameliorate rat acute renal failure by differentiation into renal tubular epithelial-like cells. Int J Mol Med 22: 325-332, 2008.

22. Räsänen $K$ and Vaheri A: Activation of fibroblasts in cancer stroma. Exp Cell Res 316: 2713-2722, 2010.

23. Augsten M, Hägglöf C, Olsson E, et al: CXCL14 is an autocrine growth factor for fibroblasts and acts as a multi-modal stimulator of prostate tumor growth. Proc Natl Acad Sci USA 106: 3414-3419, 2009 .
24. Thiery JP: Epithelial-mesenchymal transitions in development and pathologies. Curr Opin Celll Biol 15: 740-746, 2003.

25. Allan GJ, Beattie $J$ and Flint DJ: Epithelial injury induces an innate repair mechanism linked to cellular senescence and fibrosis involving IGF-binding protein-5. J Endocrinol 199: 155-164, 2008.

26. Kalluri R and Weinberg RA: The basics of epithelial-mesenchymal transition. J Clin Invest 119: 1420-1428, 2009.

27. Massagué J: TGFbeta in cancer. Cell 134: 215-230, 2008.

28. Padua D and Massagué J: Roles of TGFbeta in metastasis. Cell Res 19: 89-102, 2009.

29. Buijs JT, Henriquez NV, van Overveld PG, van der Horst G, ten Dijke P and van der Pluijm G: TGF-beta and BMP7 interactions in tumour progression and bone metastasis. Clin Exp Metastasis 24: 609-617, 2007

30. Yang J and Weinberg RA: Epithelial-mesenchymal transition: at the crossroads of development and tumor metastasis. Dev Cell 14: 818-829, 2008.

31. Christiansen JJ and Rajasekaran AK: Reassessing epithelial to mesenchymal transition as a prerequisite for carcinoma invasion and metastasis. Cancer Res 66: 8319-8326, 2006.

32. Tsukamoto H, Shibata K, Kajiyama H, Terauchi M, Nawa A and Kikkawa F: Irradiation-induced epithelial-mesenchymal transition (EMT) related to invasive potential in endometrial carcinoma cells. Gynecol Oncol 107: 500-504, 2007.

33. SI PH and HU QG: Progress of RNA interfere in gene therapy for oral tumor. Int J Stomatol 134: 281-283, 2007.

34. Berdiel-Acer M, Bohem ME, López-Doriga A, et al: Hepatic carcinoma-associated fibroblasts promote an adaptative response in colorectal cancer cells that inhibit proliferation and apoptosis: nonresistant cells die by nonapoptotic cell death. Neoplasia 13 931-946, 2011.

35. Vega S, Morales AV, Ocaña OH, Valdés F, Fabregat I and Nieto MA: Snail blocks the cell cycle and confers resistance to cell death. Genes Dev 18: 1131-1143, 2004.

36. Morel AP, Lièvre M, Thomas C, Hinkal G, Ansieau S and Puisieux A: Generation of breast cancer stem cells through epithelial-mesenchymal transition. PLoS One 3: e2888, 2008.

37. Mani SA, Guo W, Liao MJ, et al: The epithelial-mesenchymal transition generates cells with properties of stem cells. Cell 133 704-715, 2008

38. Thiery JP, Aclogue H, Huang RY and Nieto MA: Epithelialmesenchymal transitions in development and disease. Cell 139: 871-890, 2009

39. Jeter CR, Badeaux M, Choy G, et al: Functional evidence that the self-renewal gene NANOG regulates human tumor development. Stem Cells 27: 993-1005, 2009.

40. Lu Y, Futtner C, Rock JR, Xu X, Whitworth W, Hogan BL and Onaitis MW: Evidence that SOX2 overexpression is oncogenic in the lung. PLoS One 5: el11022, 2010.

41. Qiao B, Chen Z, Hu F, Tao Q and Lam AK: BMI-1 activation is crucial in hTERT-induced epithelial-mesenchymal transition of oral epithelial cells. Exp Mol Pathol 95: 57-61, 2013. 